\title{
Isolation Procedure for CP E. coli from Caeca Samples under Review towards an Increased Sensitivity
}

\author{
Natalie Pauly ${ }^{1}$, Yvonne Klaar ${ }^{1}$, Tanja Skladnikiewicz-Ziemer ${ }^{1}$, Katharina Juraschek ${ }^{1}$, Mirjam Grobbel ${ }^{1} \mathbb{D}$, \\ Jens André Hammerl ${ }^{1}{ }^{(}$, Lukas Hemmers ${ }^{2}$, Annemarie Käsbohrer ${ }^{1,3}{ }^{\oplus}$, Stefan Schwarz ${ }^{4,5}{ }^{\oplus}$, Diana Meemken ${ }^{6}{ }^{(1)}$, \\ Bernd-Alois Tenhagen ${ }^{1}$ and Alexandra Irrgang ${ }^{1, *}$ (i)
}

Citation: Pauly, N.; Klaar, Y.;

Skladnikiewicz-Ziemer, T.; Juraschek,

K.; Grobbel, M.; Hammerl, J.A.;

Hemmers, L.; Käsbohrer, A.; Schwarz,

S.; Meemken, D.; et al. Isolation

Procedure for CP E. coli from Caeca

Samples under Review towards an Increased Sensitivity. Microorganisms 2021, 9, 1105. https://doi.org/ 10.3390/microorganisms 9051105

Academic Editor: Labrini

V. Athanasiou

Received: 30 March 2021

Accepted: 14 May 2021

Published: 20 May 2021

Publisher's Note: MDPI stays neutral with regard to jurisdictional claims in published maps and institutional affiliations.

Copyright: (c) 2021 by the authors. Licensee MDPI, Basel, Switzerland. This article is an open access article distributed under the terms and conditions of the Creative Commons Attribution (CC BY) license (https:// creativecommons.org/licenses/by/ $4.0 /)$.
1 German Federal Institute for Risk Assessment (Bundesinstitut für Risikobewertung), D-10589 Berlin, Germany; natalie_pauly@hotmail.com (N.P.); Yvonne.Klaar@bfr.bund.de (Y.K.); Tanja.Skladnikiewicz-Ziemer@bfr.bund.de (T.S.-Z.); Katharina.juraschek@bfr.bund.de (K.J.); mirjam.grobbel@bfr.bund.de (M.G.); Jens-Andre.Hammerl@bfr.bund.de (J.A.H.); annemarie.kaesbohrer@bfr.bund.de (A.K.); bernd-alois.tenhagen@bfr.bund.de (B.-A.T.)

2 State Office for Health and Social Affairs Berlin, D-10639 Berlin, Germany; lukas.hemmers@lageso.berline.de

3 Unit for Veterinary Public Health and Epidemiology, University of Veterinary Medicine, AT-1210 Vienna, Austria

4 Institute of Microbiology and Epizootics, Freie Universität Berlin, D-14163 Berlin, Germany; Stefan.Schwarz@fu-berlin.de

5 FAO Reference Centre for Antimicrobial Resistance, Freie Universität Berlin, D-14163 Berlin, Germany

6 Institute of Food Safety and Food Hygiene, Freie Universität Berlin, D-14163 Berlin, Germany; diana.meemken@fu-berlin.de

* Correspondence: Alexandra.irrgang@bfr.bund.de

Abstract: Due to the increasing reports of carbapenemase-producing Enterobacteriaceae (CPE) from livestock in recent years, the European Reference Laboratory for Antimicrobial Resistances (EURLAR) provided a protocol for their recovery from caecum and meat samples. This procedure exhibited limitations for the detection of CPE with low carbapenem MIC values. Therefore, it was modified by a second, selective enrichment in lysogeny broth with cefotaxime (CTX $1 \mathrm{mg} / \mathrm{L}$ ) and with meropenem (MEM $0.125 \mathrm{mg} / \mathrm{L}$ ) at $37^{\circ} \mathrm{C}$ under microaerophilic conditions. By Real-time PCR, these enrichments are pre-screened for the most common carbapenemase genes. Another adaptation was the use of in-house prepared MacConkey agar with MEM and MEM+CTX instead of commercial selective agar. According to the EURL-method, we achieved 100\% sensitivity and specificity using the in-house media instead of commercial agar, which decreased the sensitivity to $\sim 75 \%$. Comparing the method with and without the second enrichment, no substantial influence on sensitivity and specificity was detected. Nevertheless, this enrichment has simplified the CPE-isolation regarding the accompanying microbiota and the separation of putative colonies. In conclusion, the sensitivity of the method can be increased with slight modifications.

Keywords: Isolation; carbapenemase; CPE detection; selective media; specificity; sensitivity

\section{Introduction}

The World Health Organization defines carbapenems as 'High Priority Critically Important Antimicrobials' for human medicine [1]. This is due to their role in the treatment of severe human infections with multidrug-resistant bacteria. Up to now, in European countries carbapenems are not licensed for use in veterinary medicine. Nevertheless, carbapenems may be used in specific circumstances in companion animals [2,3]. In recent years, an increasing number of carbapenem resistant enterobacteria, including carbapenemaseproducers, have been reported [4]. Interestingly, the reports are not only limited to human medicine [4-6] as carbapenem-resistant bacteria (i.e., E. coli, Salmonella enterica subsp. enterica) have also been detected repeatedly in environmental or animal samples $[4,5]$. 
Besides various chromosomally encoded mechanisms (i.e., changes in membrane permeability, efflux pumps), resistance to carbapenems is often mediated by degrading enzymes called carbapenemases that can hydrolyze carbapenems and most other $\beta$-lactams [7]. Typically, the corresponding carbapenemase genes are located on mobile (integrative and conjugative) genetic elements (i.e., plasmids) [8,9]. They exhibit different mechanisms of action (i.e., serine-based (i.e., KPC, OXA) and zinc-catalysed carbapenemases (i.e., NDM, VIM)) $[10,11]$. Carbapenemases usually show a broad activity against most $\beta$-lactams and are typically associated with resistance determinants against other antimicrobial classes often leading to a drastic reduction of therapeutic options in case of infections [12,13]. In the European Union (EU), OXA, VIM, NDM, and KPC carbapenemases are most frequently detected in human clinical settings [5,14,15]. In 2012, the first carbapenemase-producing (CP) E. coli was detected in livestock [16], and voluntary monitoring of CP E. coli in the EU member states along with the obligatory monitoring on extended spectrum $\beta$-lactamase (ESBL)/AmpC $\beta$-lactamases producing E. coli (CID 2013/652/EU) [17] was introduced. In 2021, through new legislation, the specific monitoring for carbapenem resistant $E$. coli became mandatory (CID 2020/1729/EU). For selective CP E. coli detection, the isolation method provided by the European Reference Laboratory for Antimicrobial Resistances (EURL-AR) (https:/ / www.eurl-ar.eu / protocols.aspx, accessed on December 2019) is recommended. The method is based on a 1:10 non-selective enrichment of the samples in buffered peptone water (BPW), which needs to be inoculated onto suitable selective agars (i.e., the commercial chromID ${ }^{\circledR}$ CARBA agar). Several studies described the chromID ${ }^{\circledR}$ CARBA agar as one of the most sensitive and specific chromogenic media for the detection of CP Enterobacteriaceae (CPE) in clinical samples in human medicine [18-21]. In Germany, this agar represents one of the most commonly used selective media for CPE monitoring purposes [22]. By using a highly sensitive molecular pre-screening of enrichment cultures (i.e., by real-time PCR), laborious microbiological investigations might be avoided, and further efforts can focus on the recovery of CPE from presumptive-positive samples.

Unfortunately, for some CPE detected in German livestock the EURL-AR detection method failed in recent years [23,24]. For example, the isolate 17-AB02384 was not detected within the monitoring of CP E. coli but was recovered in the monitoring on ESBL/AmpC $\beta$-lactamase producing E. coli from the same sample [24]. Thus, we identified the necessity to improve the isolation method for CP E. coli from livestock samples, especially from caecum content. Difficulties in the microbiological processing of caeca samples are the growth of an accompanying microbiota, the low CPE concentrations, and the considerably lower MIC values of CPE from animal origin in comparison to human isolates [22]. Here, we present comparative analyses on the performance of the EURL-method with a number of alterations potentially leading to an improvement of the CPE detection and recovery rate. The focus of this study was on the recovery of CP E. coli from pig caecum, as the majority of the recently described CP E. coli from German livestock originate from this matrix. Therefore, samples were spiked and blinded to calculate the sensitivity and specificity of the approaches, which included different selective agars.

\section{Materials and Methods}

\subsection{Pre-Studies}

Initial experiments were carried out to determine the growth and the survival of CPE in feces. Therefore, the survival rate (colony forming units (cfu)/g pig feces) of seven different CPE (four VIM-1 producing E. coli, two VIM-1 producing Salmonella (S.) Infantis and one NDM-1 producing $S$. Corvallis) was investigated over a period of 10 days. For the alternative method to be tested, spiked samples were prepared. For this, the seven CPE were inoculated each in lysogeny broth (LB) supplemented with $1 \mathrm{mg} / \mathrm{L}$ cefotaxime (CTX) $(\mathrm{LB}+\mathrm{CTX})$ and were incubated at $37^{\circ} \mathrm{C}$ for $16-18 \mathrm{~h}$. After incubation, $10 \mu \mathrm{L}$ of the suspension was transferred into LB and was additionally incubated at $37^{\circ} \mathrm{C}$ with $180 \mathrm{rpm}$ until an optical density $\left(\mathrm{OD}_{600 \mathrm{~nm}}\right)$ of 0.5 was reached. The suspensions were centrifuged for $10 \mathrm{~min}$ at $4000 \mathrm{rpm}$ and resuspended in $0.9 \%$ saline solution $(w / v)$. Thereafter, $5 \mathrm{~mL}$ of the 
solution $\left(\sim 10^{8} \mathrm{cfu} / \mathrm{mL}\right)$ was applied to $45 \mathrm{~g}$ feces and homogenized for $1 \mathrm{~min}$ in a BagMixer $400 S W$ (Interscience, Wiesbaden, Germany). The prepared samples were stored at $4-6{ }^{\circ} \mathrm{C}$. Every $24 \mathrm{~h}, 1 \mathrm{~g}$ of the spiked samples was diluted in $0.9 \%$ saline solution to $10^{-6}$. Aliquots of $100 \mu \mathrm{L}$ of the $10^{-5}$ and the $10^{-6}$ dilutions were plated out onto selective MacConkey agar (McC) supplemented with $0.125 \mathrm{mg} / \mathrm{L}$ meropenem (MEM) $(\mathrm{McC}+\mathrm{MEM})$ and $1 \mathrm{mg} / \mathrm{L}$ CTX and MEM (McC + CTX + MEM), and the cfu were counted after incubation for 20-24 h at $37^{\circ} \mathrm{C}$.

To extend the time until sample processing in the laboratory, a potential improvement of the CPE survival in fecal samples was tested by adding stabilizing substances. Trehalose [25,26], glycerol $[27,28]$, and sodium chloride $[29,30]$ were tested as potential additives for the improvement of the recovery rate of the seven CPE in pig feces. Previous studies reported on the protective characteristics or the enhancive influence of these substances for the recovery and growth of Enterobacteriaceae [25-30]. Therefore, the described previous experiments were repeated by adding $1 \mathrm{~mL}$ of the supplement (trehalose, glycerol, and sodium acid) to $9 \mathrm{~g}$ of the caeca sample. The samples were thoroughly mixed for $1 \mathrm{~min}$ and a decimal dilution series up to $10^{-6}$ in $0.9 \%$ saline solution was prepared. Afterwards, $100 \mu \mathrm{L}$ of the $10^{-5}$ and the $10^{-6}$ dilution were applied onto selective McC + CTX + MEM, incubated overnight at $37^{\circ} \mathrm{C}$, and $\mathrm{cfu} / \mathrm{g}$ were determined.

The influence of different liquid media (Mossel medium [31] and LB) on CPE growth was tested to determine the most appropriate culture medium for the second enrichment step. Furthermore, possible influences of a sublethal concentration of MEM $(0.008 \mu \mathrm{g} / \mathrm{mL})$ [32] and the addition of $\mathrm{ZnSO}_{4}(70 \mu \mathrm{g} / \mathrm{mL})$ [33] to the two liquid media were tested. Gullberg et al. (2014) [34] reported that sublethal antimicrobial concentrations are able to induce bacteria to retain plasmids and express corresponding genes.

Moreover, the growth of a CP E. coli and a CP Salmonella in LB + CTX and LB + MEM under aerobic and microaerobic conditions were compared. For spiking the samples, each $\mathrm{CPE}$ was inoculated in $4 \mathrm{~mL} \mathrm{BPW}$ and incubated at $37^{\circ} \mathrm{C}$ overnight. Thereafter, $50 \mu \mathrm{L}$ of the culture was transferred to BPW and incubated at $37^{\circ} \mathrm{C}$ and $180 \mathrm{rpm}$ until an optical density $\left(\mathrm{OD}_{600} \mathrm{~nm}\right)$ of 1.0 was reached. From this suspension, $1 \mathrm{~mL}$ was added to $9 \mathrm{~g}$ fresh caeca sample. Thereafter, each $1 \mathrm{~mL}$ of this mix was transferred to $9 \mathrm{~mL} \mathrm{LB}+\mathrm{CTX}$ and incubated at $37^{\circ} \mathrm{C}$ either under aerobic or under microaerobic conditions for $18 \mathrm{~h}$. After incubation, $100 \mu \mathrm{L}$ of the sample were plated four times onto McC + CTX + MEM and cfu were counted after $20-24 \mathrm{~h}$ of incubation at $37^{\circ} \mathrm{C}$. Potential differences induced by the two incubation conditions were assessed through a two-sided Wilcoxon signed rank test for independent samples.

\subsection{Method Comparison}

\subsubsection{Bacterial Strains}

In total, twelve CP E. coli were used for the evaluation of both isolation methods and the comparison of the different selective media. These isolates cover a wide range of carbapenemase genes $\left(b l a_{\mathrm{VIM}-1} ; b l a_{\mathrm{GES}-5} ; b l a_{\mathrm{KPC}-2} ; b l a_{\mathrm{NDM}-1}\right.$ and $\left.b l a_{\mathrm{OXA}-48}\right)$ (Supplemental Material Table S1). As reference for quality assurance concerning the selectivity for E. coli of the media, the E. coli ATCC 25922 was used. In all three approaches, six caeca samples were CPE-negative and inoculated with the E. coli ATCC 25922. In each of the other twelve caeca samples, one of the twelve CP E. coli was inoculated with a concentration of $100 \mathrm{cfu} / \mathrm{mL}$ each.

\subsubsection{Sample Preparation for the Method Comparison}

All twelve CP E. coli and the E. coli control strain ATCC 25922 were inoculated in $4 \mathrm{~mL} \mathrm{BPW}$ and incubated at $37^{\circ} \mathrm{C}$ overnight. After incubation, $50 \mu \mathrm{L}$ of the overnight culture was transferred to fresh BPW and incubated at $37^{\circ} \mathrm{C}$ at $180 \mathrm{rpm}$ until an optical density $\left(\mathrm{OD}_{600} \mathrm{~nm}\right)$ of 1 was reached. For each CP E. coli, $9 \mathrm{~g}$ fresh caeca sample was artificially contaminated with $1 \mathrm{~mL}$ of the $10^{-5}$ diluted bacterial suspension. For the control strain, six caeca samples were spiked. All spiked caeca samples were homogenized by 
thorough mixing for $1 \mathrm{~min}$. After sample preparation, the samples were blinded by a non-participating person. All 18 samples were analyzed by two methods (Figure 1) as described below. First, $1 \mathrm{~g}$ of each spiked sample was added to $9 \mathrm{~mL}$ BPW and incubated at $37 \pm 2{ }^{\circ} \mathrm{C}$ for $16-18 \mathrm{~h}$. Thereafter, the samples were separately subjected to investigations using the two different isolation procedures. For reliable interpretation, the 18 blinded samples were investigated with both methods in three independent approaches.

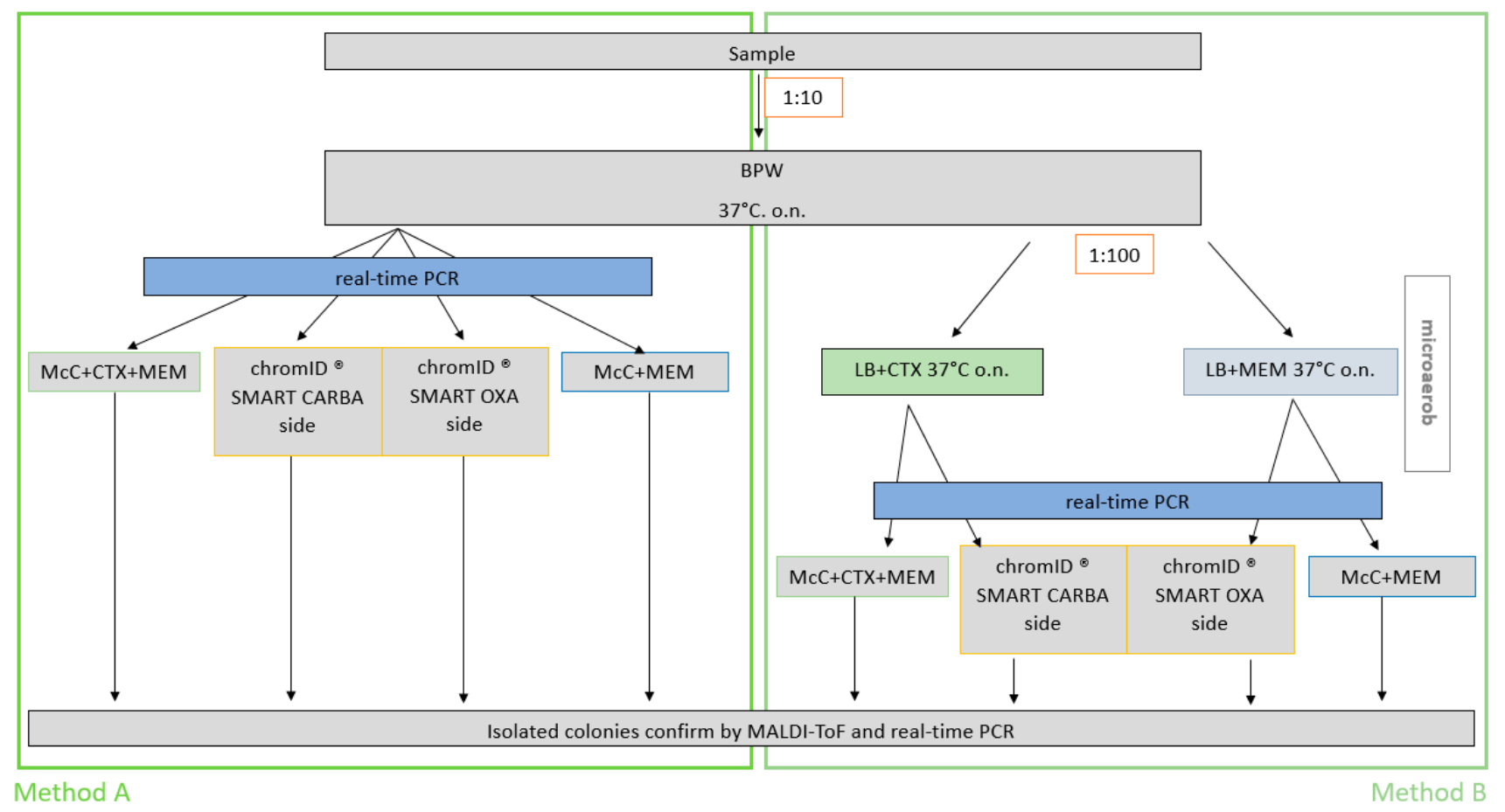

Figure 1. The whole procedure of method A and method B to compare both and to validate the agar plates. BPW $=\mathrm{Buffered}$ Peptone Water; McC + CTX + MEM = MacConkey Agar supplemented with $1 \mathrm{mg} / \mathrm{L}$ cefotaxim and 0.125 mg/L meropenem; $\mathrm{McC}+\mathrm{MEM}=$ MacConkey Agar supplemented with $0.125 \mathrm{mg} / \mathrm{L}$ meropenem; LB + CTX = lysogeny broth supplemented with $1 \mathrm{mg} / \mathrm{L}$ cefotaxim; LB+MEM = lysogeny broth supplemented with $0.125 \mathrm{mg} / \mathrm{L}$ meropenem.

\subsubsection{Isolation and Detection Methods}

Method A represents the EURL-protocol for isolation of ESBL, AmpC and CP E. coli from caeca, (https: / / www.eurl-ar.eu/protocols.aspx, accessed in December 2019). For the method comparison, the commercial chromID ${ }^{\circledR}$ CARBA SMART agar (bioMérieux, Nürtingen, Germany) was used. This agar is composed of the CARBA agar and the OXA agar in equal parts. Therefore, the EURL-protocol was adapted by applying $5 \mu \mathrm{L}$ on a half agar plate instead of $10 \mu \mathrm{L}$ on a full agar plate. This adaption allowed the agar validation as part of the method comparison $\left(5 \mu \mathrm{L}\right.$ per half chromID ${ }^{\circledR}$ CARBA SMART agar plate, $10 \mu \mathrm{L}$ per a whole $\mathrm{McC}$ agar plate) (Figure 2). Additionally, a Real-Time PCR step was added after the enrichment in BPW (described below).

Method $\mathrm{B}$ represents the modified version of the isolation procedure. Therein, nonselective enrichment in BPW has been maintained, followed by a second selective enrichment step. For that purpose, $10 \mu \mathrm{L}$ of the BPW pre-enrichment has been applied to $10 \mathrm{~mL} \mathrm{LB}+\mathrm{CTX}$ and $10 \mathrm{~mL} \mathrm{LB}+\mathrm{MEM}$. Both enrichments were incubated at $37 \pm 2{ }^{\circ} \mathrm{C}$ for 16-18 $\mathrm{h}$ under microaerophilic conditions. Thereafter, $5 \mu \mathrm{L}$ of the $\mathrm{LB}+\mathrm{CTX}$ enrichment was inoculated on the CARBA side, and $5 \mu \mathrm{L}$ of the LB + MEM enrichment on the OXA side of the chromID ${ }^{\circledR}$ CARBA SMART plate (Figure 1). In addition, $10 \mu \mathrm{L}$ of LB + CTX or LB + MEM were inoculated on in-house selective agars McC + CTX + MEM or McC + MEM, respectively. Each sample was stroked on each agar in duplicate (six plates per sample). Plates were incubated for $16-18 \mathrm{~h}$ at $37 \pm 2{ }^{\circ} \mathrm{C}$. Subsequently, one colony with 
E. coli typical colony morphology was picked from each plate and enriched in $4 \mathrm{~mL} \mathrm{LB}$ + MEM. The species of each colony was confirmed by PCR and MALDI-ToF. Confirmed colonies were incubated for $16-18 \mathrm{~h}$ at $37 \pm 2{ }^{\circ} \mathrm{C}$ in $\mathrm{LB}$ and $500 \mu \mathrm{L}$ aliquots were stored in $800 \mu \mathrm{L}$ of $80 \%$ glycerol for further analysis. Samples spiked with OXA-producers were assessed as positive if growth was observed on the chromID ${ }^{\circledR}$ OXA side, likewise on McC + MEM. For the other CP E. coli, the sample was regarded positive if growth was observed at least on chromID ${ }^{\circledR}$ CARBA side or on $\mathrm{McC}+\mathrm{CTX}+\mathrm{MEM}$.

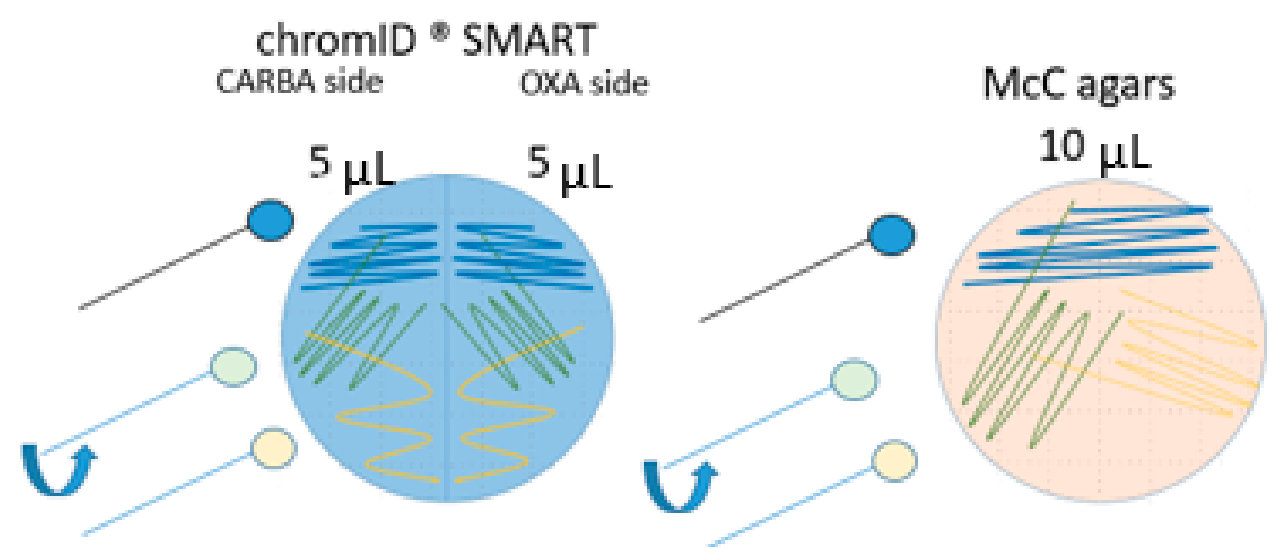

Figure 2. Spreading the sample on the plate. The first spread (blue, first third of the plate) was performed by using a $10 \mu \mathrm{L}$-loop. A second spread (green, second third of the plate) was performed by another $10 \mu \mathrm{L}$-loop, which was turned the last third of the spread (yellow).

To confirm the validity of the results, the whole experiment was independently repeated three times. Moreover, to ensure that personal handling had no influence, both methods were carried out by two different people per approach. Moreover, each agar type was used in duplicate and deviations (i.e., colony morphology) were noted. If at least one colony could be isolated and confirmed from one of the duplicates, the sample was rated positive for the corresponding method and agar type.

\subsubsection{Typing}

Antimicrobial Susceptibility-Testing (AST)

The antimicrobial susceptibility of the isolates (Supplemental Material Table S1) was determined by broth microdilution using defined antimicrobial substances and concentrations from the harmonized EU panel following Commission Implementing Decision (CID) 2013/652/EU [plates EUVSEC \& EUVSEC2; TREK Diagnostic Systems (Thermo Fisher Scientific, Schwerte, Germany)]. AST was conducted according to the EN ISO20776-1:2006 [15] and MIC values were interpreted based on EUCAST definitions (www.eucast.org, November 2013) using epidemiological cut-off values fixed in CID 2013/652/2013. The strain ATCC 25922 was used for quality control. All isolates were tested twice. First, before using them for artificial contamination and second, after their successful recovery from the samples to assess possible changes in their antimicrobial resistance patterns.

\section{MALDI-ToF MS}

One putative colony of CP E. coli of each agar plate (six per sample) was chosen for species confirmation by MALDI-ToF MS. As matrix $\alpha$-Cyano-4-hydroxycinnamic acid (HCCA, Bruker, USA) was used, and analyses were performed by MALDI Microflex Biotyper (Bruker Daltonics, Bremen, Germany) as recommended by the manufacturers.

Molecular Typing of CP E. coli

Molecular detection of the carbapenemase genes was carried out to confirm the presence/absence of $b l a_{\mathrm{GES}}, b l a_{\mathrm{KPC}}, b l a_{\mathrm{NDM}}, b l a_{\mathrm{OXA}-48}$ and $b l a_{\mathrm{VIM}}$ in enrichment cultures and in presumptive colonies. Both sample enrichments and presumptive colonies were 
used for extraction of template DNA by heat treatment of bacterial suspensions. For this extraction, $800 \mu \mathrm{L}$ were centrifuged by $10,000 \times g$ for $5 \mathrm{~min}$. The cell pellet was resuspended in $300 \mu \mathrm{L}$ double distilled water and boiled for $10 \mathrm{~min}$ at $99{ }^{\circ} \mathrm{C}$. Thereafter, the samples were cooled on ice and centrifuged for $2 \mathrm{~min}$ at $16,000 \times g$. Finally, $255 \mu \mathrm{L}$ of the supernatant was mixed with $45 \mu \mathrm{L}$ trehalose for stable storage at $-20^{\circ} \mathrm{C}$. Real-time PCR was performed on a Bio-Rad CFX system. Primers and probes were adapted from Swayne et al. (2011) [35] and van der Zee et al. (2014) [36]. Amplification was conducted using Biozym qPCR Mastermix (Biozym Scientific, Oldendorf, Germany) and the following conditions: Initially 2 min at $95^{\circ} \mathrm{C}, 30$ cycles with $5 \mathrm{~s}$ at $95^{\circ} \mathrm{C}$ and $60 \mathrm{~s}$ at $60{ }^{\circ} \mathrm{C}$ for product amplification.

\subsubsection{Statistics}

Results of both detection methods were expressed in percent (positive samples divided by all tested samples). The EURL-method and the modified method were compared in terms of their diagnostic accuracy indicated by sensitivity, specificity, false discovery and omission rates, and overall diagnostic accuracy. At this point, the blinding of the samples was dissolved. If the detected CP E. coli were confirmed using MALDI-ToF MS and real-time PCR, a sample was classified as positive. The sensitivity was expressed as the proportion of the positive samples correctly identified. The specificity was calculated as the proportion of the samples correctly identified as negative among all negative samples. False discovery rates were calculated as the proportion of all false positive samples among all positive samples. Likewise, false omission rates were calculated as the proportion of all false negative samples among all negative samples. Overall diagnostic accuracy was calculated as the proportion of all tests that gave a correct result. All estimates as well as their corresponding exact 95\% confidence intervals (Clopper-Pearson 95\% confidence intervals as precision measures honoring small sample sizes) were estimated using the epiR package in $\mathrm{R}$ [37].

\section{Results}

\subsection{Modification of the EURL-Protocol for Isolation of CP E. coli from Caeca Samples}

In order to determine the number of bacteria that survived the prevailing condition in the pig feces, the recovery rate of the bacteria in feces was determined after storage at $6^{\circ} \mathrm{C}$. Based on the cfu counts, a reduction of $2 \log$ units within $24 \mathrm{~h}$ was detected in pig feces samples stored at $6{ }^{\circ} \mathrm{C}$. The addition of glycerin, sodium chloride, and trehalose to the feces samples did not result in a positive effect on the CPE survival rate (Figure 3).

In a first step, the suitability of two liquid media (LB- or Mossel-medium) as basis of this second enrichment were compared. The Mossel-medium was developed especially for Enterobacteriaceae [31], whereas LB-medium is a nutrient-rich microbial broth used for the cultivation of E. coli. No differences in the recovery rate were observed in the direct comparison of LB- or Mossel-medium as second enrichment (data not shown). As LB is a common medium used in many laboratories, it was used for further investigations.

The addition of sublethal conditions of $\mathrm{ZnSO}_{4}$ and $\mathrm{MEM}$ was intended to activate potentially present resistance mechanisms in the liquid culture so that the corresponding strains may have a growth advantage when subsequently plated onto the selective solid media. However, the slight selection pressure did not result in a better recovery rate. Therefore, we used the second enrichment in LB + CTX as previously described [23]. To isolate the OXA-48 producers as well, we tested another second enrichment in LB + MEM. Enterobacteriaceae are described as facultative anaerobes [38]. Therefore, the target species can survive in both aerobic and anaerobic environments, in contrast to some representatives of the accompanying microbiota. The microaerophilic incubation led to a median increase in isolated CP E. coli from $7 \mathrm{cfu} / \mathrm{mL}$ with aerobic incubation to $26.5 \mathrm{cfu} / \mathrm{mL}$ (Wilcoxon test, $\mathrm{W}=0, p=<0.05, n=8$ ). Likewise, the median number of CP Salmonella increased from $81 \mathrm{cfu} / \mathrm{mL}$ with aerobic incubation to $144.5 \mathrm{cfu} / \mathrm{mL}$ (Wilcoxon test, $\mathrm{W}=0, p=<0.05, n=8)$. 


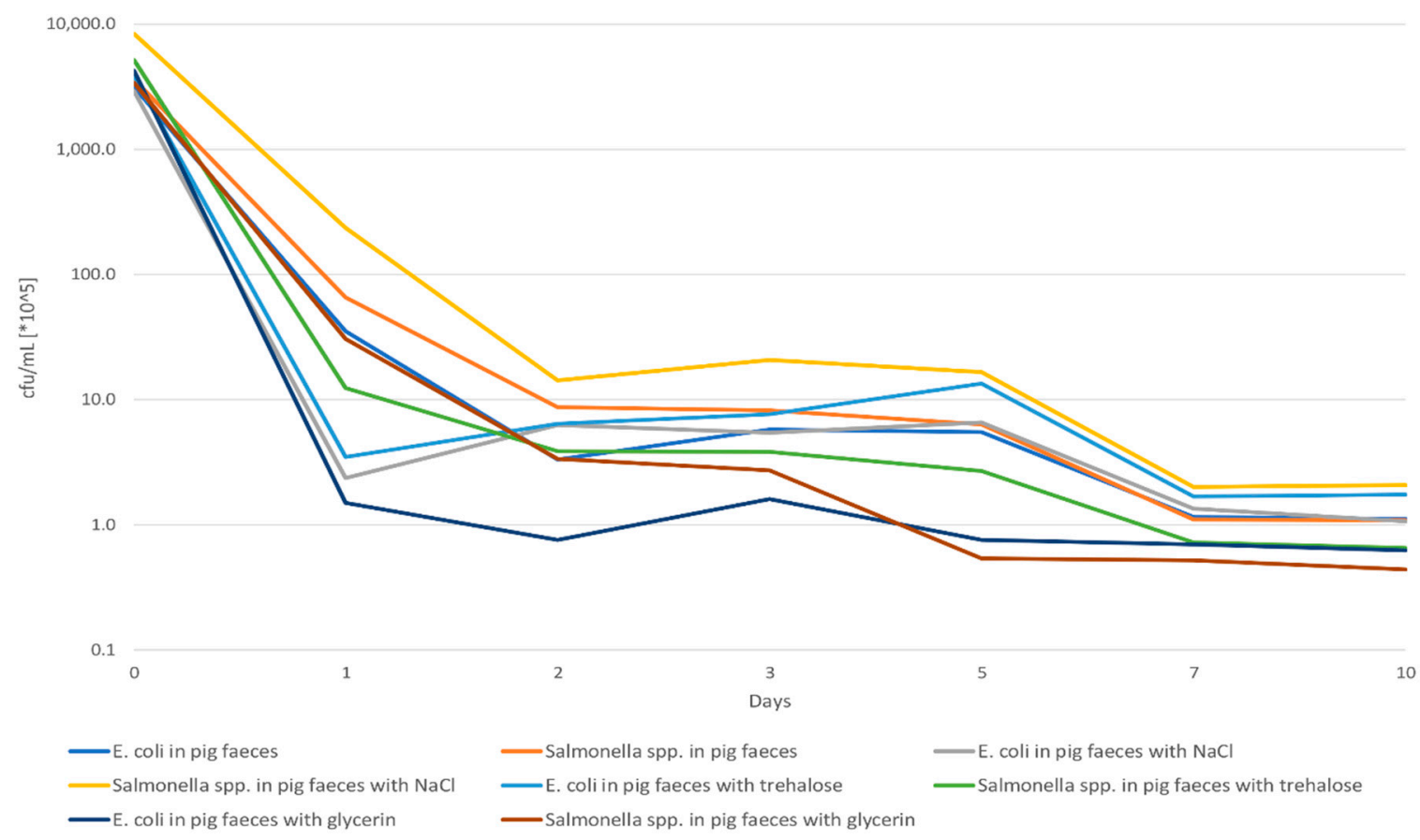

Figure 3. The decreasing number of CPE (E. coli and Salmonella each in average) in pig faeces with and without some additives over ten days.

Based on the above-mentioned observations, the procedure of the method was modified to promote the growth of $\mathrm{CPE}$ and to reduce the amount of accompanying microbiota (Figure 4). The modified procedure includes a first, non-selective enrichment in BPW, followed by two parallel selective enrichments (LB + CTX and LB + MEM) incubated under microaerophilic conditions. Aliquots $(10 \mu \mathrm{L})$ of the second enrichment were applied onto McC agar supplemented either with MEM or CTX + MEM. In the following chapter, this modified method was compared to the EURL-method.

3.2. The Comparison of the Modified Method with the EURL-Method for Isolation of the CP E. coli from Caeca Samples

To determine the performance of the modified method (method B), its diagnostic accuracy was compared to the reference method of the EURL-AR, the official protocol for isolation of ESBL, AmpC and CP E. coli from caeca samples (method A). The complete procedure of the comparison and validation is schematically illustrated in Figure 1. In the following, the results of the three repetitions were considered together (sample size $n=54$ ) for each method and agar type (Table 1).

Table 1. Summarized results on the repeated isolation of CPE from the spiked fecal samples (calculation by combining the OXA and CARBA- site of the ChromID ${ }^{\circledR}$ SMART CARBA agars and of the McC + CTX + MEM and the McC + MEM plate of the in-house agar). Method B is the modified method. Corresponding exact $95 \%$ confidence intervals are provided in brackets.

\begin{tabular}{ccccc}
\hline & $\begin{array}{c}\text { Method A }+ \\
\text { ChromID } \\
\text { CARB }\end{array}$ & $\begin{array}{c}\text { Method A }+ \\
\text { in-house agars }\end{array}$ & $\begin{array}{c}\text { Method B + } \\
\text { in-house agars }\end{array}$ & $\begin{array}{c}\text { Method B + } \\
\text { ChromID } \\
\text { CARBA agars }\end{array}$ \\
\hline Sensitivity & $75(57.8-87.9)$ & $100(90.2-100)$ & $86.1(70.5-95.3)$ & $66.7(49-81.4)$ \\
\hline Specificity & $100(81.5-100)$ & $100(81.5-100)$ & $100(81.5-100)$ & $100(81.5-100)$ \\
\hline False discovery rate & $0(0-12.8)$ & $0(0-9.7)$ & $0(0-11.2)$ & $0(0-14.2)$ \\
\hline False omission rate & $33.3(16.5-54)$ & $0(0-18.5)$ & $21.7(7.5-43.7)$ & $40(22.7-59.4)$ \\
\hline Accuracy & $83.3(70.7-92.1)$ & $100(93.4-100)$ & $90.7(79.7-96.9)$ & $77.8(64.4-88)$ \\
\hline
\end{tabular}




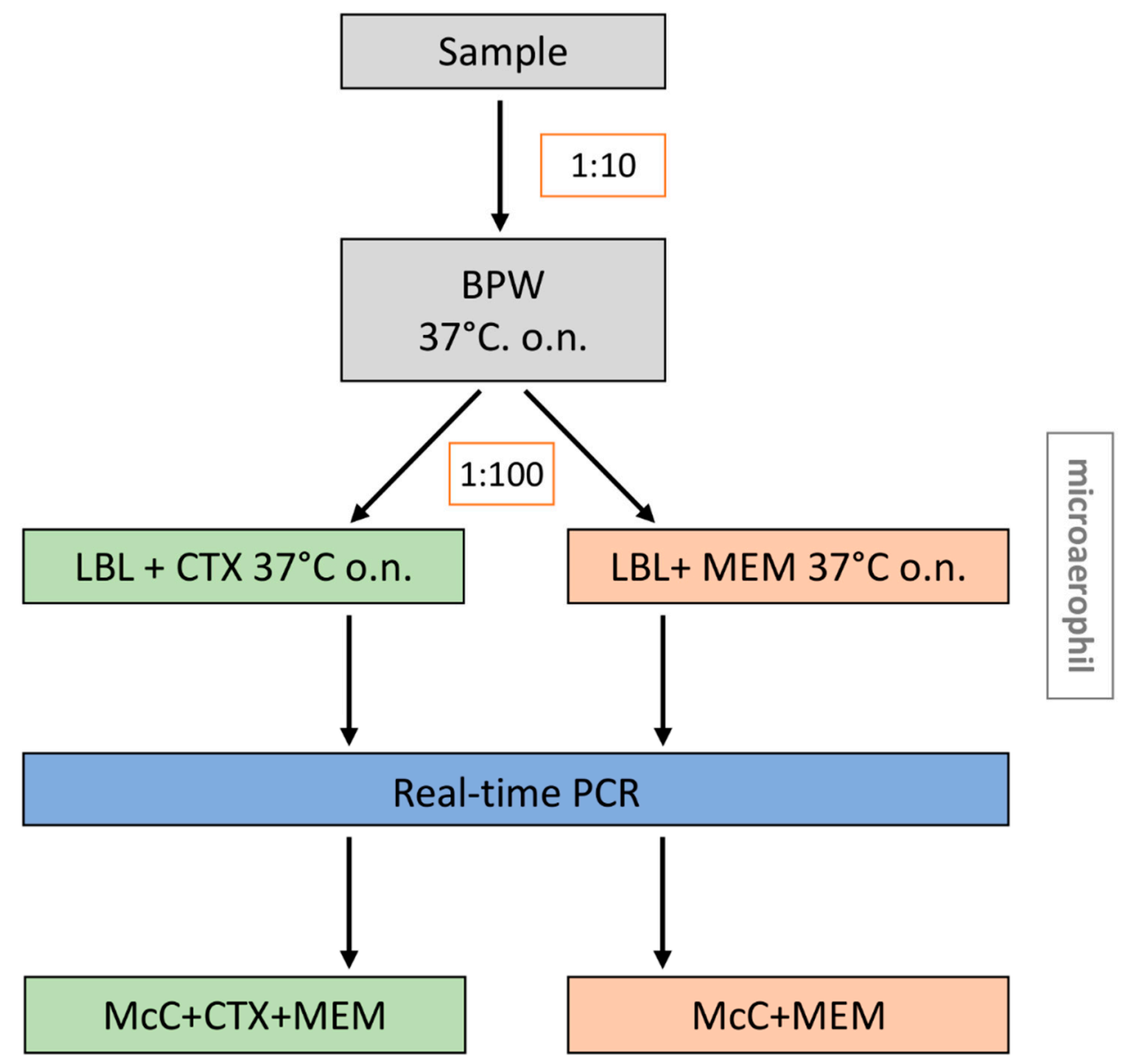

Figure 4. The final step-by-step guidance of the modified method (method B). BPW = Buffered Peptone Water; McC + CTX + MEM = MacConkey Agar supplemented with $1 \mathrm{mg} / \mathrm{L}$ cefotaxim and $0.125 \mathrm{mg} / \mathrm{L}$ meropenem; McC + MEM = MacConkey Agar supplemented with $0.125 \mathrm{mg} / \mathrm{L}$ meropenem; LB + CTX = lysogeny broth supplemented with $1 \mathrm{mg} / \mathrm{L}$ cefotaxim; $\mathrm{LB}+\mathrm{MEM}=$ lysogeny broth supplemented with $0.125 \mathrm{mg} / \mathrm{L}$ meropenem.

Comparison of diagnostic accuracy measures show that the use of method A in combination with the in-house agar led to a significantly higher sensitivity, relative to the use of method A with the chromID ${ }^{\circledR}$ SMART CARBA agar. In addition to this overall evaluation, the results for the individual approaches are given in Supplemental Material Table S2. The best CPE detection was achieved by using the original EURL-protocol in combination with in-house prepared McC + MEM and McC + CTX + MEM agar plates. All negative samples $(n / \mathrm{N})$ were correctly recognized, and CP E. coli were successfully recovered from each of the artificially contaminated samples $(n / \mathrm{N})$. This corresponds to a $100 \%$ sensitivity and specificity. The sensitivity decreased to $75 \%$ when the in-house agar plates were replaced by the commercial chromID ${ }^{\circledR}$ CARBA agar. However, specificity remained at $100 \%$. The modified method (B) reached a sensitivity of $86.1 \%$ when using the in-house agar and a sensitivity of $66.7 \%$ when using the commercial agar. However, all negative samples $(n / N)$ were also correctly identified leading to a specificity of $100 \%$. The added value of this modified method is the simplification of the isolation of putative colonies that are difficult to isolate. This could be shown by counting the agar plates (108 per agar type and method) with and without accompanying microbiota (i.e., Pseudomonas (P.) putida, P. monteilli, P. aeruginosa, Proteus mirabilis) (Table 2). Using the EURL-procedure in combination with the chromID ${ }^{\circledR}$ CARBA agar, on $44.5 \%(48 / 108)$ of the agar plates growth of accompanying microbiota was observed. That was reduced to $39.8 \%(43 / 108)$ using the modified method with the chromID ${ }^{\circledR}$ CARBA agar. In addition, for the chromID ${ }^{\circledR}$ OXA agar, a reduction of accompanying microbiota from $23.2 \%(25 / 108)$ of the plates by 
using the EURL-procedure to $15.7 \%(17 / 108)$ using the modified method was observed. Using the in-house agars and the EURL-procedure, accompanying microbiota was observed on $37.0 \%(40 / 108)$ of the McC + MEM plates and on $33.4 \%(36 / 108)$ of the McC + CTX + MEM plates. By changing to the modified method, we detected accompanying microbiota on $29.6 \%(32 / 108)$ of the McC + MEM plates and on $36.1 \%(39 / 108)$ of the McC + CTX + MEM plates.

Table 2. Validation of the selective agars by counting the number of plates with growing of accompanying microbiota for each group per used method. McC + CTX + MEM = MacConkey Agar supplemented with $1 \mathrm{mg} / \mathrm{L}$ cefotaxim and $0.125 \mathrm{mg} / \mathrm{L}$ meropenem; McC + MEM = MacConkey Agar supplemented with $0.125 \mathrm{mg} / \mathrm{L}$ meropenem.

\begin{tabular}{|c|c|c|c|c|}
\hline & $\begin{array}{c}\text { ChromID }^{\circledR} \text { SMART } \\
\text { CARBA Side }\end{array}$ & $\begin{array}{c}\text { ChromID }^{\circledR} \text { SMART } \\
\text { OXA Side }\end{array}$ & McC + MEM & $\mathrm{McC}+\mathrm{CTX}+\mathrm{MEM}$ \\
\hline Method A & $\begin{array}{c}48 / 108 \\
(44.45 \%)\end{array}$ & $\begin{array}{c}25 / 108 \\
(23.15 \%)\end{array}$ & $\begin{array}{c}40 / 108 \\
(37.04 \%)\end{array}$ & $\begin{array}{c}36 / 108 \\
(33.36 \%)\end{array}$ \\
\hline Method B & $\begin{array}{c}43 / 108 \\
(39.82 \%)\end{array}$ & $\begin{array}{c}17 / 108 \\
(15.74 \%)\end{array}$ & $\begin{array}{c}32 / 108 \\
(29.63 \%)\end{array}$ & $\begin{array}{c}39 / 108 \\
(36.14 \%)\end{array}$ \\
\hline
\end{tabular}

\subsection{Molecular Detection of the Carbapenemase Genes}

As previously described, the multiplex real-time PCR was used to pre-screen the enrichments (BPW, LB + CTX, and LB + MEM). Discrepancies were detected between the PCR and the results of the culture-based isolation. Comparing the PCR results with the true status of the samples, a sensitivity of $61.1 \%$ and a specificity of $94.4 \%$ was calculated for the molecular screening of the heat-treated BPW enrichments for carbapenemase genes. Fourteen out of thirty-six positive samples were not correctly identified. One of the 18 negative samples was incorrectly identified as positive for the bla $\mathrm{GES}_{\text {. }}$

When considering the screening of both second enrichments (results listed in Table 3), just two of the false negatives remained false negative, and the false positive sample remained as well. Therefore, the PCR achieved a sensitivity and a specificity of $94.4 \%$ by using both second enrichments as DNA template. Moreover, the real-time PCR showed erroneous simultaneous detection of bla $\mathrm{NDM}_{\mathrm{N}}$ and bla $\mathrm{VIM}_{\mathrm{V}}$ in various samples $(9.2 \%)$ that were only blaviM-positive. However, a high cp-valuefor bla NDM was detected, suggesting low amplification. The results for the weekly approaches are given in Supplemental Material Table S3.

Table 3. Accumulated results on the weekly detection of carbapenemase genes from the spiked fecal samples. BPW was used for the first enrichment, LB + CTX and LB + MEM were used parallel as second enrichments. Corresponding exact $95 \%$ confidence intervals are provided in brackets.

\begin{tabular}{ccccc}
\hline & BPW & LB + CTX & LB + MEM & Both LB Enrichments \\
\hline Sensitivity & $61.1(43.5-76.9)$ & $83.3(67.2-93.6)$ & $58.3(40.8-74.4)$ & $94.4(81.3-99.3)$ \\
\hline Specifity & $94.4(72.7-99.6)$ & $94.4(72.7-99.6)$ & $100(81.5-100)$ & $94.4(72.7-99.9)$ \\
\hline False discovery rate & $4.3(0.1-21.9)$ & $3.2(0.1-16.7)$ & $0(0-16.1)$ & $2.9(0.1-14.9)$ \\
\hline False omission rate & $45.2(27.3-64)$ & $26.1(10.2-48.4)$ & $45.4(28.1-63.6)$ & $10.5(1.3-33.1)$ \\
\hline Accuracy & $72.2(58.4-83.5)$ & $87.0(75.1-94.7)$ & $72.2(58.4-83.5)$ & $94.4(84.6-98.8)$ \\
\hline
\end{tabular}

\section{Discussion}

\subsection{Modification Steps of the Official Isolation Protocol}

All modifications were tested to reduce the amount of accompanying microbiota or to increase the recovery rate of the CPE. First, the recovery rate of the target bacteria in pig feces was determined at $6{ }^{\circ} \mathrm{C}$ and based on the bacterial growth calculated by the development of the $\mathrm{cfu} / \mathrm{g}$ every day. To increase the survival of bacteria for better isolation, several additives to the pig feces were tested. In previous studies, the addition of sodium 
chloride has been reported to support growth under difficult conditions like low $\mathrm{pH}$ and high lactate concentrations [29]. Another study evaluated trehalose for the protection of E. coli against carbon stress [25]. The absence of a positive effect of these additives in our study may be due to the lack of a clear definition of the individual influencing factors of each fecal sample, i.e., the microbiota and other factors, which could have an influence on the nature of the samples. It should be noted here that the detection of CPE was increasingly challenging over the investigated period. One major challenge was the differentiation of $\mathrm{CPE}$ from increasing amounts of accompanying flora. Therefore, we recommend a timely processing of samples, preferably within $24 \mathrm{~h}$ after collection as this was an important factor for reliable detection of CP E. coli in feces and food matrices.

After estimating bacterial survival, other options for improved detection of the target bacteria were tested. To account for the low MIC values of CPE isolated from samples of animal origin [22], we considered triggering gene expression by using a sublethal concentration of MEM. Moreover, the usage of $\mathrm{ZnSO}_{4}$ to push the metallo- $\beta$-lactamases was tested. Both approaches did not improve the recovery rate. Previous experiments achieved a beneficial effect by using a second selective enrichment step. This enrichment had been conducted in LB+CTX [23]. As OXA-48 producers are not reliably able to survive the presence of other $\beta$-lactams, like CTX, we considered another parallel second enrichment using LB+MEM $[39,40]$. The usefulness of the combination of both enrichments was also proven by the sensitivity of $94.4 \%$ of the Real-Time PCR.

Further, we exchanged the commercial selective agar chromID ${ }^{\circledR}$ CARBA agar with an in-house prepared McC + CTX and McC + CTX + MEM. Our results support a previous study on the suitability of chromID ${ }^{\circledR}$ CARBA agar for detecting CPE with only slightly reduced susceptibility to carbapenems [22]. Within this study, it is presumed that the concentration of different ingredients and thus the selectivity is too high for CPE originating from animals or food. CPE from livestock often exhibit lower MIC values for the respective carbapenems than clinical human isolates [22,24]. Our previous study investigated the use of chromID ${ }^{\circledR}$ CARBA after the end of the shelf life expecting a possible decrease in antimicrobial concentration. As the selective agar is not specified in the EURL-AR reference method (https: / / www.eurl-ar.eu/protocols.aspx, December 2019), the change to adjusted agar might be easy adaptable. In this study, we analyzed the use of an in-house McC agar, supplemented with MEM and MEM+CTX, and commercial agar for the detection of CPE from feces. The experiments were carried out with fresh prepared in-house agars (two weeks). However, the comparison in this study is limited to only one commercial selective agar. For commercial agars, a wide variation regarding their suitability for the isolation of CPE from different samples has been reported $[18,20,41,42]$. For the chromID ${ }^{\circledR}$ CARBA agar, sensitivity in the literature ranges from 30 to $100 \%$, and specificity from 50 to $100 \%$. Still, the chromID ${ }^{\circledR}$ CARBA agar is described as one of the most sensitive media $[18,20,41,42]$ and is therefore commonly used by German laboratories in the monitoring according to CID 2013/652/EU.

\subsection{The Comparison of Both Microbiological Methods}

Although the isolation of $\mathrm{CPE}$ from livestock and food is rare in monitoring programs, the number of reports on successful recovery is increasing in recent years [6]. The isolation methods described differ from direct plating to different incubation conditions, supplements, and enrichments $[23,43,44]$. These non-harmonized detection methods in research projects in the animal sector complicate a realistic assessment of the occurrence of CPE in livestock. The EURL-AR has developed and provided a harmonized method for CP E. coli isolation from meat and caeca samples, which is meant to be used in monitoring according to CID 2013/652/EU. However, it is currently rarely used beyond the EU monitoring programs. Several publications reported on difficulties in detecting CPE with the EURLmethod [22-24]. We observed a sensitivity of $75 \%$ and specificity of $100 \%$ for the EURL-AR protocol with a commonly used commercial agar. This implies that $25 \%$ of CPE-positive samples were not recognized, which demonstrates the need for an improvement of the 
reference method. Our results indicate that using the validated EURL-protocol in combination with the non-commercial McC agar supplemented with $0.125 \mathrm{mg} / \mathrm{L} \mathrm{MEM}$, and with $0.125 \mathrm{mg} / \mathrm{L}$ MEM and $1 \mathrm{mg} / \mathrm{L}$ CTX significantly improved the sensitivity compared to all other approaches. Selective enrichment under microaerobic conditions did not achieve the desired effect. This microaerobic incubation was promising because of the ability of Enterobacteriaceae to grow under this condition, in contrast to e.g., Aeromonads, which are often part of the accompanying microbiota [38]. Other options to favor Enterobacteriaceae over other bacteria have been investigated. An incubation temperature of $44{ }^{\circ} \mathrm{C}$ was described in the report on the validation of selective $\mathrm{McC}$ agar supplemented with $1 \mathrm{mg} / \mathrm{L}$ CTX for monitoring of ESBL- and AmpC producing E. coli in meat and caeca samples [45] and in a previous study of Irrgang et al. 2019 [23]. The authors recognized that the growth of CP Enterobacteriaceae other than CP E. coli might also be inhibited [23]. In terms of harmonization, a method should be capable of also detecting other bacterial species, as all CPE are of interest regarding the distribution of the resistances [6]. Our study focused on E. coli as the EU monitoring for ESBL/pAmpC and carbapenemases targets this species.

The reported problems during detection of $\mathrm{CPE}$-containing samples indicate a low occurrence of CPE in samples of animal origin [23,24]. Another rarely discussed reason for reduced detection of CPE from feces and caecum could be that the bacteria in this environment have lost the ability to grow on routine media. It is described that E. coli, Klebsiella pneumoniae, Salmonella enterica serovar Typhimurium, and other human/zoonotic pathogens could enter a distinct state, a so-called viable but non-culturable (VBNC) state $[46,47]$. In combination with the physical stress exerted by caeca samples, the transition to a VBNC status would be useful for the survival of CPE [47]. Overall, we need to be able to detect positive samples and to isolate these $\mathrm{CPE}$ for a further characterization and risk assessment.

Another approach to improve sensitivity might be a more sensitive molecular screening method. Using pre-screening by PCR, efforts can focus on the cultivation of the isolates from PCR-positive samples. This would decrease the costs and the personal time necessary for the microbiological isolation. The multiplex Real-Time PCR applied in this study showed good results when screening the second enrichment (a sensitivity and specificity of $94.4 \%$ ). It might be possible that the use of a quantitative Real-Time PCR will provide more reliable results as a direct increase of the bacterial growth can be detected and used for adapting the time points for selective cultivation. The Real-Time PCR performed was not effective when applied on the initial BPW enrichment. To avoid the isolation step of a target bacterium and to immediately analyze the entire sample, the option of metagenomics becomes more attractive. Metagenomics would allow a simultaneous identification and typing of CPE and other pathogens [47]. It would be a powerful tool for monitoring purposes and might be considered in the long term to become part of the routine method. Currently, this method is too expensive for routine use and requires additional bioinformatic expertise.

\section{Conclusions}

After various approaches to optimize the recovery rate of CPE in pig feces, it could be concluded that potential additives did not have a positive effect. However, a positive effect can be achieved by processing the samples in a timely manner. Moreover, our results confirmed the influence of the chosen media. Typically used commercial agar plates are optimized for the isolation of CPE from human clinical samples with usually high MIC values for carbapenems. Therefore, we recommend the EURL-method with a selective medium corresponding to the searched target bacterium. A second enrichment under microaerophilic conditions reduced the accompanying microbiota, but the sensitivity of the whole procedure was decreased from $100 \%$ to $86.1 \%$. The PCR-screening from a second enrichment showed good results. Therefore, we recommend the modified method if a presumptive CP E. coli cannot be isolated. However, a sensitive method is essential to avoid an underestimation of the CPE occurrence in livestock in Europe. 
Supplementary Materials: The following are available online at https: / www.mdpi.com/article/ 10.3390/microorganisms9051105/s1, Supplemental Material Table S1: Detailed information about the used CPE. Supplemental Material Table S2: The repeated isolation of CPE from the spiked faecal samples. Supplemental Material Table S3: Summarized Real-time PCR results on the repeated detection of carbapenemase genes from the spiked faecal samples.

Author Contributions: Data curation, N.P.; formal analysis, L.H., S.S., and A.K.; investigation, N.P., Y.K., T.S.-Z., K.J., and M.G.; project administration, A.I. and M.G.; visualization, N.P.; writingoriginal draft, N.P., J.A.H., and A.I.; writing-review and editing, K.J., Y.K., J.A.H., M.G., L.H., A.K., B.-A.T., S.S., D.M., and A.I. All authors have read and agreed to the published version of the manuscript.

Funding: This work was financially supported by a grant of the German Federal Institute for Risk Assessment (43-001 and 43-002). The work of Natalie Pauly was supported by the European Joint Project (EJP) IMPART funded by the European Union's Horizon 2020 research and innovation programme under Grant Agreement No 773830. The work of Stefan Schwarz is supported by the German Federal Ministry of Education and Research (BMBF) under project number and 01KI2009D as part of the Research Network Zoonotic Infectious Diseases.

Institutional Review Board Statement: Not applicable.

Informed Consent Statement: Not applicable.

Data Availability Statement: MDPI: Research Data Policies.

Acknowledgments: We thank the laboratory team (Britta Lesniewsky, Anja Seeger, Silke Jahn, Romy Fuhrmeister, and Silvia Schmoger) of the NRL-AR for excellent technical assistance. We further acknowledge the support of the participating company for the provision of the sample material.

Conflicts of Interest: The authors declare no conflict of interest.

\section{References}

1. WHO. Critically Important Antimicrobials for Human Medicine, 6th ed.; World Health Organization: Geneva, Switzerland, 2019.

2. Madec, J.Y.; Haenni, M.; Nordmann, P.; Poirel, L. Extended-spectrum $\beta$-lactamase/AmpC- and carbapenemase-producing Enterobacteriaceae in animals: A threat for humans? Clin. Microbiol. Infect. 2017, 23, 826-833. [CrossRef] [PubMed]

3. Aworh, M.K.; Kwaga, J.; Okolocha, E.; Mba, N.; Thakur, S. Prevalence and risk factors for multi-drug resistant Escherichia coli among poultry workers in the Federal Capital Territory, Abuja, Nigeria. PLoS ONE 2019, 14, e0225379. [CrossRef]

4. EFSA/ECDC. The European Union summary report on antimicrobial resistance in zoonotic and indicator bacteria from hu-mans, animals and food in 2016. EFSA J. 2018, 16, e05182.

5. Köck, R.; Daniels-Haardt, I.; Becker, K.; Mellmann, A.; Friedrich, A.; Mevius, D.; Schwarz, S.; Jurke, A. Carbapenem-resistant Enterobacteriaceae in wildlife, food-producing, and companion animals: A systematic review. Clin. Microbiol. Infect. 2018, 24, 1241-1250. [CrossRef]

6. Taneja, N.; Sharma, M. Antimicrobial resistance in the environment: The Indian scenario. Indian J. Med. Res. 2019, 149, 119-128. [CrossRef]

7. Miriagou, V.; Cornaglia, G.; Edelstein, M.; Galani, I.; Giske, C.G.; Gniadkowski, M.; Malamou-Lada, E.; Martinez-Martinez, L.; Navarro, F.; Nordmann, P.; et al. Acquired car-bapenemases in Gram-negative bacterial pathogens: Detection and surveillance issues. Clin. Microbiol. Infect. 2010, 16, 112-122. [CrossRef]

8. Partridge, S.R.; Kwong, S.M.; Firth, N.; Jensen, S.O. Mobile Genetic Elements Associated with Antimicrobial Resistance. Clin. Microbiol. Rev. 2018, 31, e00088-17. [CrossRef] [PubMed]

9. Botelho, J.; Roberts, A.P.; León-Sampedro, R.; Grosso, F.; Peixe, L. Carbapenemases on the move: It's good to be on ICEs. Mob. DNA 2018, 9, 37. [CrossRef]

10. Palzkill, T. Metallo- $\beta$-lactamase structure and function. Ann. N. Y. Acad. Sci. 2013, 1277, 91-104. [CrossRef]

11. Salahuddin, P.; Kumar, A.; Kha, A.U. Structure, function of serine and Metallo- $\beta$-lactamases and their Inhibitors. Curr. Protein. Pept. Sci. 2018, 19, 130-144. [CrossRef]

12. Bush, K. Past and present perspectives on $\beta$-lactamases. Antimicrob. Agents Chemother. 2018, 62, e01076-18. [CrossRef]

13. Woodford, N.; Wareham, D.W.; Guerra, B.; Teale, C. Carbapenemase-producing Enterobacteriaceae and non-Enterobacteriaceae from animals and the environment: An emerging public health risk of our own making? J. Antimicrob. Chemother. 2014, 69, 287-291. [CrossRef]

14. Grundmann, H.; Glasner, C.; Albiger, B.; Aanensen, D.M.; Tomlinson, C.T.; Andrasević, A.T.; Cantón, R.; Carmeli, Y.; Friedrich, A.W.; Giske, C.G.; et al. Occurrence of carbapenemase-producing Klebsiella pneumoniae and Escherichia coli in the European survey of carbapenemase-producing Enterobacteriaceae (EuSCAPE): A prospective, multinational study. Lancet Infect. Dis. 2017, 17, 153-163. [CrossRef] 
15. EFSA and ECDC. The European Union summary report on antimicrobial resistance in zoonotic and indicator bacteria from humans, animals and food in 2017. EFSA J. 2019, 17, e05598.

16. Fischer, J.; Rodriguez, I.; Schmoger, S.; Friese, A.; Roesler, U.; Helmuth, R.; Guerra, B. Escherichia coli producing VIM-1 car-bapenemase isolated on a pig farm. J. Antimicrob. Chemother. 2012, 67, 1793-1795. [CrossRef]

17. Schrijver, R.; Stijntjes, M.; Rodríguez-Baño, J.; Tacconelli, E.; Rajendran, N.B.; Voss, A. Review of antimicrobial resistance surveillance programmes in livestock and meat in EU with focus on humans. Clin. Microbiol. Infect. 2018, 24, 577-590. [CrossRef]

18. Simner, P.J.; Gilmour, M.W.; DeGagne, P.; Nichol, K.; Karlowsky, J.A. Evaluation of Five Chromogenic Agar Media and the Rosco Rapid Carb Screen Kit for Detection and Confirmation of Carbapenemase Production in Gram-Negative Bacilli. J. Clin. Microbiol. 2014, 53, 105-112. [CrossRef]

19. Wilkinson, K.M.; Winstanley, T.G.; Lanyon, C.; Cummings, S.P.; Raza, M.W.; Perry, J.D. Comparison of Four Chromogenic Culture Media for Carbapenemase-Producing Enterobacteriaceae. J. Clin. Microbiol. 2012, 50, 3102-3104. [CrossRef]

20. Vrioni, G.; Daniil, I.; Voulgari, E.; Ranellou, K.; Koumaki, V.; Ghirardi, S.; Kimouli, M.; Zambardi, G.; Tsakris, A. Comparative evaluation of a prototype chromogenic medium (ChromID CARBA) for detecting carbapenemase-producing Enterobacte-riaceae in surveillance rectal swabs. J. Clin. Microbiol. 2012, 50, 1841-1846. [CrossRef]

21. Papadimitriou-Olivgeris, M.; Bartzavali, C.; Christofidou, M.; Bereksi, N.; Hey, J.; Zambardi, G.; Spiliopoulou, I. Performance of chromID ${ }^{\circledR}$ CARBA medium for carbapenemases-producing enterobacteriaceae detection during rectal screening. Eur. J. Clin. Microbiol. Infect. Dis. 2014, 33, 35-40. [CrossRef]

22. Pauly, N.; Hammerl, J.A.; Grobbel, M.; Tenhagen, B.-A.; Käsbohrer, A.; Bisenius, S.; Fuchs, J.; Horlacher, S.; Lingstädt, H.; Mauermann, U.; et al. ChromID ${ }^{\circledR}$ CARBA Agar Fails to Detect Carbapenem-Resistant Enterobacteriaceae with Slightly Reduced Susceptibility to Carbapenems. Front. Microbiol. 2020, 11, 1678. [CrossRef]

23. Irrgang, A.; Tenhagen, B.-A.; Pauly, N.; Schmoger, S.; Kaesbohrer, A.; Hammerl, J.A. Characterization of VIM-1-Producing E. coli Isolated from a German Fattening Pig Farm by an Improved Isolation Procedure. Front. Microbiol. 2019, 10, 2256. [CrossRef]

24. Pauly, N.; Hammerl, J.A.; Schwarz, S.; Grobbel, M.; Meemken, D.; Malorny, B.; Tenhagen, B.-A.; Käsbohrer, A.; Irrgang, A. Co-occurrence of the blaVIM-1 and blaSHV-12 genes on an IncHI2 plasmid of an Escherichia coli isolate recovered from German livestock. J. Antimicrob. Chemother. 2021, 76, 531-533. [CrossRef] [PubMed]

25. Algara, M.M.; Kuczyńska-Wiśnik, D.; Dębski, J.; Stojowska-Swędrzyńska, K.; Sominka, H.; Bukrejewska, M.; Laskowska, E. Trehalose protects Escherichia coli against carbon stress manifested by protein acetylation and aggregation. Mol. Microbiol. 2019, 112, 866-880. [CrossRef] [PubMed]

26. Kuczyńska-Wiśnik, D.; Stojowska, K.; Matuszewska, E.; Leszczyńska, D.; Algara, M.M.; Augustynowicz, M.; Laskowska, E. Lack of intracellular trehalose affects formation of Escherichia coli persister cells. Microbiol. Spectr. 2015, 161 Pt 4, 786-796. [CrossRef]

27. Achá, S.; Kühn, I.; Mbazima, G.; Colque-Navarro, P.; Möllby, R. Changes of viability and composition of the Escherichia coli flora in faecal samples during long time storage. J. Microbiol. Methods 2005, 63, 229-238. [CrossRef]

28. Hollander, D.H.; Nell, E.E. Improved Preservation of Treponema pallidum and Other Bacteria by Freezing with Glycerol. Appl. Microbiol. 1954, 2, 164-170. [CrossRef] [PubMed]

29. Jordan, D. Sodium chloride enhances recovery and growth of acid-stressed E. coli O157:H7. Lett. Appl. Microbiol. 2001, 32, 312-315. [CrossRef]

30. Mañas, P.; Pagan, R.; Leguérinel, I.; Condon, S.; Mafart, P.; Sala, F. Effect of sodium chloride concentration on the heat resistance and recovery of Salmonella typhimurium. Int. J. Food Microbiol. 2001, 63, 209-216. [CrossRef]

31. Mossel, D.A.; Harrewijn, G.A.; Zadelhoff, C.F.N.-V. Standardization of the selective inhibitory effect of surface active compounds used in media for the detection of Enterobacteriaceae in foods and water. Health Lab. Sci. 1974, 11, $260-267$.

32. Andersson, D.I.; Hughes, D. Microbiological effects of sublethal levels of antibiotics. Nat. Rev. Genet. 2014, 12, 465-478. [CrossRef] [PubMed]

33. Nordmann, P.; Girlich, D.; Poirel, L. Detection of Carbapenemase Producers in Enterobacteriaceae by Use of a Novel Screening Medium. J. Clin. Microbiol. 2012, 50, 2761-2766. [CrossRef] [PubMed]

34. Gullberg, E.; Albrecht, L.M.; Karlsson, C.; Sandegren, L.; Andersson, D.I. Selection of a Multidrug Resistance Plasmid by Sublethal Levels of Antibiotics and Heavy Metals. mBio 2014, 5, e01918-14. [CrossRef] [PubMed]

35. Swayne, R.; Ludlam, H.; Shet, V.; Woodford, N.; Curran, M. Real-time TaqMan PCR for rapid detection of genes encoding five types of non-metallo- (class A and D) carbapenemases in Enterobacteriaceae. Int. J. Antimicrob. Agents 2011, 38, 35-38. [CrossRef] [PubMed]

36. Van Der Zee, A.; Roorda, L.; Bosman, G.; Fluit, A.C.; Hermans, M.; Smits, P.H.; Van Der Zanden, A.G.; Witt, R.T.; Van Coppenraet, L.E.B.; Stuart, J.C.; et al. Multi-centre evaluation of real-time multiplex PCR for detection of carbapenemase genes OXA-48, VIM, IMP, NDM and KPC. BMC Infect. Dis. 2014, 14, 27. [CrossRef]

37. R Core Team. R: A Language and Environment for Statistical Computing; R Foundation for Statistical Computing: Vienna, Austria, 2020; Available online: http:/ /www.R-project.org/ (accessed on 11 March 2021).

38. Octavia, S.; Lan, R. The Family Enterobacteriaceae. In The Prokaryotes; Springer: Berlin/Heidelberg, Germany, $2014 ;$ pp. 225-286. ISBN 9783642389221.

39. Poirel, L.; Potron, A.; Nordmann, P. OXA-48-like carbapenemases: The phantom menace. J. Antimicrob. Chemother. 2012, 67, 1597-1606. [CrossRef]

40. Codjoe, F.S.; Donkor, E.S. Carbapenem Resistance: A Review. Med. Sci. 2017, 6, 1. [CrossRef] 
41. Viau, R.; Frank, K.M.; Jacobs, M.R.; Wilson, B.; Kaye, K.; Donskey, C.J.; Perez, F.; Endimiani, A.; Bonomo, R.A. Intestinal Carriage of Carbapenemase-Producing Organisms: Current Status of Surveillance Methods. Clin. Microbiol. Rev. 2015, 29, 1-27. [CrossRef]

42. Brand, P.; Gobeli, S.; Perreten, V. Pathotyping and antibiotic resistance of porcine enterovirulent Escherichia coli strains from Switzerland (2014-2015). Schweiz Arch Tierheilkd 2017, 159, 373-380. [CrossRef]

43. Mo, S.S.; Urdahl, A.M.; Madslien, K.; Sunde, M.; Nesse, L.L.; Slettemeås, J.S.; Norström, M. What does the fox say? Monitoring antimicrobial resistance in the environment using wild red foxes as an indicator. PLoS ONE 2018, 13, e0198019. [CrossRef]

44. Hasman, H.; Agersø, Y.; Cavaco, L.M. Validation of selective MacConkey agar plates supplemented with $1 \mathrm{mg} / \mathrm{L}$ cefo-taxime for monitoring of ESBL- and AmpC-producing E. coli in meat and caeca samples. LABORATORY PROTOCOL Authored by the EURL-AR. 2017. Available online: https:/ / www.eurl-ar.eu/CustomerData/Files/Folders/21-protocols/390_protocol-forvalidation-of-macconkey-and-ctx-agar-plates-final03112017.pdf (accessed on November 2013).

45. Xu, H.-S.; Roberts, N.; Singleton, F.L.; Attwell, R.W.; Grimes, D.J.; Colwell, R.R. Survival and viability of nonculturableEscherichia coli andVibrio cholerae in the estuarine and marine environment. Microb. Ecol. 1982, 8, 313-323. [CrossRef] [PubMed]

46. Li, L.; Mendis, N.; Trigui, H.; Oliver, J.D.; Faucher, S.P. The importance of the viable but non-culturable state in human bacterial pathogens. Front. Microbiol. 2014, 5, 258. [CrossRef]

47. Grützke, J.; Malorny, B.; Hammerl, J.A.; Busch, A.; Tausch, S.H.; Tomaso, H.; Deneke, C. Fishing in the Soup-Pathogen Detection in Food Safety Using Metabarcoding and Metagenomic Sequencing. Front. Microbiol. 2019, 10, 1805. [CrossRef] [PubMed] 